\title{
Nonequilibrium Entropy Conservation and the Transport Equations of Mass, Momentum, and Energy
}

\author{
Michael H. Peters
}

check for updates

Citation: Peters, M.H

Nonequilibrium Entropy

Conservation and the Transport

Equations of Mass, Momentum, and Energy. Energies 2021, 14, 2196.

https://doi.org/10.3390/en14082196

Academic Editors: Yousef Haseli and Andrea Mariscotti

Received: 9 February 2021

Accepted: 9 April 2021

Published: 14 April 2021

Publisher's Note: MDPI stays neutral with regard to jurisdictional claims in published maps and institutional affiliations.

Copyright: (c) 2021 by the author Licensee MDPI, Basel, Switzerland. This article is an open access article distributed under the terms and conditions of the Creative Commons Attribution (CC BY) license (https:/ / creativecommons.org/licenses/by/ $4.0 /)$.
Department of Chemical and Life Science Engineering, Virginia Commonwealth University, 601 West Main St., Richmond, VA 23284, USA; mpeters@vcu.edu

\begin{abstract}
Nonequilibrium statistical mechanics or molecular theory has put the transport equations of mass, momentum and energy on a firm or rigorous theoretical foundation that has played a critical role in their use and applications. Here, it is shown that those methods can be extended to nonequilibrium entropy conservation. As already known, the "closure" of the transport equations leads to the theory underlying the phenomenological laws, including Fick's Law of Diffusion, Newton's Law of Viscosity, and Fourier's Law of Heat. In the case of entropy, closure leads to the relationship of entropy flux to heat as well as the Second Law or the necessity of positive entropy generation. It is further demonstrated how the complete set of transport equations, including entropy, can be simplified under physically restrictive assumptions, such as reversible flows and local equilibrium flows. This analysis, in general, yields a complete, rigorous set of transport equations for use in applications. Finally, it is also shown how this basis set of transport equations can be transformed to a new set of nonequilibrium thermodynamic functions, such as the nonequilibrium Gibbs' transport equation derived here, which may have additional practical utility.
\end{abstract}

Keywords: theory of entropy conservation; energy efficiency; energy applications; second law

\section{Introduction}

Nonequilibrium entropy conservation for open systems is often the "forgotten" transport equation in the analysis and applications of the conservation laws to physico-chemical systems. Irving and Kirkwood [1] developed the conservation equations of mass, momentum, and energy from the molecular level, beginning with the Liouville equation or the fundamental equation of classical statistical mechanics, putting most of transport phenomena on a firm theoretical foundation. This includes the theory behind the phenomenological laws, such as Newton's Law of Viscosity, Fourier's Law of Heat, and Fick's Law of Diffusion. As shown here, Irving and Kirkwood's methodology can be extended to include entropy conservation and the Second Law. Importantly, as originally shown by Jaynes [2,3], nonequilibrium entropy conservation and the Second Law only follows willful approximations of the underlying molecular probability density functions. In fact, it is well-known that the Liouville equation itself cannot support the principle of entropy increase and the Second Law without approximations, which we have termed "Liouville's Paradox" [4]. From a practical level, the Second Law sets limits on the amounts of work and heat that are possible for a particular system, as well as "unallowed" states that still may satisfy the principles of mass and energy conservation but not exist. Additionally, simplifications are often invoked in order to obtain expected transport behavior under "ideal" conditions, including the categories of local equilibrium flows, reversible flows, and inviscid flows. As shown here, the molecular approach to entropy conservation allows us to clarify those simplifying conditions in the context of the complete set of transport equations giving internal consistency to their use in applications and analysis.

This paper is broken down into three main sections in order to accomplish this goal. The Liouville equation is presented and used to review the development of the conservation 
equations of mass, momentum and energy according to the Irving and Kirkwood (IK) approach. Then, we turn our attention to the derivation of the Second Law or entropy conservation in open systems, and finally look at special cases across the complete set of transport equations in order to provide a basis for applications that seek the behavior of systems in simplified forms. It is to be noted that no attempt is made here to review the multitude of approaches to nonequilibrium entropy treatments in the literature; rather, we base our development on the Liouville equation or the foundational equation of classical statistical mechanics.

\section{Materials and Methods}

The Liouville Equation and the Transport Equations of Mass, Momentum, and Energy

We begin with the many-body Liouville equation for particle number preserving systems written as

$$
\frac{\partial f_{N}}{\partial t}=-\sum_{i=1}^{N}\left[\frac{\mathbf{p}_{i}}{m} \cdot \frac{\partial f_{N}}{\partial \mathbf{r}_{i}}+\mathbf{F}_{i}\left(\mathbf{r}^{N}\right) \cdot \frac{\partial f_{N}}{\partial \mathbf{p}_{i}}\right]
$$

where $f_{N}$ is the $\mathrm{N}$-particle density function, $\mathbf{r}_{i}$ and $\mathbf{p}_{i}$ are the position and momentum coordinates for the ith particle of mass $m$, and we have assumed that the force acting on particle $i$ is only a function of the position coordinates $\mathbf{r}^{N} \equiv\left(\mathbf{r}_{1}, \mathbf{r}_{2}, \ldots, \mathbf{r}_{N}\right)$. It is assumed that the $N$ particles are spin-less, and they are indistinguishable, i.e., we have a pure component system. Using the gradient of a potential, the force on molecule $i$ the can be written as

$$
\mathbf{F}_{i}=-\frac{\partial}{\partial \mathbf{r}_{i}} \Phi^{N}\left(\mathbf{r}^{N}\right)
$$

In practice, the total force or potential is expressed using the pairwise additivity approximation

$$
\mathbf{F}_{i}=-\frac{\partial}{\partial \mathbf{r}_{i}} \Phi^{N}\left(\mathbf{r}^{N}\right) \approx-\sum_{\substack{j=1 \\ j \neq i}}^{N} \frac{\partial}{\partial \mathbf{r}_{i}} \phi\left(\mathbf{r}_{i j}\right)
$$

where the pair potential $\phi\left(\mathbf{r}_{i j}\right)$ is the interaction potential between any two molecules in the system $\left(\mathbf{r}_{i j}=\mathbf{r}_{j}-\mathbf{r}_{i}\right)$.

Following, IK [1], the mass conservation equation is obtained by multiplying Equation (1) by

$$
\alpha=\sum_{k=1}^{N} \delta\left(\mathbf{r}_{k}-\mathbf{r}\right),
$$

and integrating over all phase space to obtain

$$
\frac{\partial n}{\partial t}+\frac{\partial}{\partial \mathbf{r}} \cdot\left[n \mathbf{v}_{0}\right]=0
$$

where $\mathbf{r}$ is an arbitrary locator vector and

$$
\begin{gathered}
<\alpha>=\frac{1}{N !} \sum_{k=1}^{N} \int f_{N}\left(\mathbf{r}^{N}, \mathbf{p}^{N}, t\right) d \mathbf{r}^{N} d \mathbf{p}^{N}=\frac{1}{N !} \sum_{k=1}^{N}(N-1) ! \iint \delta\left(\mathbf{r}_{k}-\mathbf{r}\right) f_{1}\left(\mathbf{r}_{k}, \mathbf{p}_{k}, t\right) d \mathbf{r}_{k} d \mathbf{p}_{k} \\
=\frac{1}{N} \sum_{k=1}^{N} \int \delta\left(\mathbf{r}_{k}-\mathbf{r}\right) n\left(\mathbf{r}_{k}, t\right) d \mathbf{r}_{k} \\
=n(\mathbf{r}, t)
\end{gathered}
$$

with the definition of the local mass average velocity, $\mathbf{v}_{0}(\mathbf{r}, t)$ :

$$
\mathbf{v}_{0}(\mathbf{r}, t) \equiv \frac{1}{n} \int\left(\frac{\mathbf{p}}{m}\right) f_{1}(\mathbf{r}, \mathbf{p}, t) d \mathbf{p}
$$


or, in terms of mass, we can obtain the so-called Continuity equation

$$
\frac{\partial \rho}{\partial t}+\frac{\partial}{\partial \mathbf{r}} \cdot\left[\rho \mathbf{v}_{0}\right]=0
$$

where $\rho=m n$

Next, the momentum conservation equation is obtained by multiplying Equation (1) by

$$
\alpha=\sum_{k=1}^{N} \mathbf{p}_{k} \delta\left(\mathbf{r}_{k}-\mathbf{r}\right)
$$

giving [1]

$$
\frac{\partial}{\partial t}\left(\rho \mathbf{v}_{0}\right)+\frac{\partial}{\partial \mathbf{r}} \cdot\left(\rho \mathbf{v}_{0} \mathbf{v}_{0}\right)=-\frac{\partial}{\partial \mathbf{r}} \cdot \mathbf{P}+\rho \frac{\mathbf{F}_{e x t}}{m}
$$

The left-hand side of Equation (9) can be expanded, and subtracting the continuity equation, Equation (8), we obtain the general momentum conservation equation

$$
\rho \frac{\partial}{\partial t} \mathbf{v}_{0}+\rho\left(\mathbf{v}_{0} \cdot \frac{\partial}{\partial \mathbf{r}}\right) \mathbf{v}_{0}=-\frac{\partial}{\partial \mathbf{r}} \cdot \mathbf{P}+\rho \frac{\mathbf{F}_{\text {ext }}}{m}
$$

where the pressure tensor $\mathbf{P}$ is defined as

$$
\mathbf{P} \equiv \mathbf{P}_{k}+\mathbf{P}_{\phi}
$$

with the kinetic contribution

$$
\mathbf{P}_{k}=m \int\left(\frac{\mathbf{p}}{m}-\mathbf{v}_{0}\right)\left(\frac{\mathbf{p}}{m}-\mathbf{v}_{0}\right) f_{1}(\mathbf{r}, \mathbf{p}, t) d \mathbf{p}
$$

and the potential contribution

$$
\mathbf{P}_{\phi}=-\frac{1}{2} n^{2}(\mathbf{r}, t) \int \tilde{g}(\mathbf{r}, \mathbf{R}, t) \frac{\mathbf{R} \mathbf{R}}{R} \frac{d \phi_{\text {int }}(R)}{d R} d \mathbf{R}
$$

where $\mathbf{R}=\mathbf{r}_{\mathbf{2}}-\mathbf{r}$ is the separation distance vector between a molecule at $\mathbf{r}$ and another molecule at $\mathbf{r}_{2}, \tilde{g}(\mathbf{r}, \mathbf{R}, t)$ is a nonequilibrium radial density function, and the pair potential $\phi_{\text {int }}(R)$ is assumed to be spherically symmetric for the sake of simplicity [1].

In order to obtain the energy conservation equation, Irving and Kirkwood [1] showed that $\alpha$ should be given by

$$
\alpha=\frac{1}{2 m} \sum_{k=1}^{N} p_{k}^{2} \delta\left(\mathbf{r}_{k}-\mathbf{r}\right)+\sum_{k=1}^{N} \phi_{\text {ext }}(\mathbf{r}, t) \delta\left(\mathbf{r}_{k}-\mathbf{r}\right)+\frac{1}{2} \sum_{j} \sum_{\substack{k \\ k \neq j}} \phi_{i n t}\left(\left|\mathbf{r}_{j}-\mathbf{r}_{k}\right|\right) \delta\left(\mathbf{r}_{k}-\mathbf{r}\right)
$$

where, $\alpha$ is the total of the kinetic, external potential $\left(\phi_{e x t}\right)$, and intermolecular potential $\left(\phi_{\text {int }}\right)$ energies evaluated at the locator vector $\mathbf{r}$ and at a time $t$. Multiplying Equation (1) by Equation (14) and integrating over all space leads to [1]

$$
\begin{gathered}
\frac{\partial}{\partial t} n\left(\bar{U}+\frac{1}{2} m v_{0}^{2}+\phi_{\text {ext }}\right)+\frac{\partial}{\partial \mathbf{r}} \cdot n \mathbf{v}_{0}\left(\bar{U}+\frac{1}{2} m v_{0}^{2}+\phi_{\text {ext }}\right) \\
=-\frac{\partial}{\partial \mathbf{r}} \cdot\left(\mathbf{v}_{0} \cdot \mathbf{P}\right)-\frac{\partial}{\partial \mathbf{r}} \cdot \mathbf{q}
\end{gathered}
$$

where we have defined the internal energy per molecule

$$
\bar{U}(\mathbf{r}, t) \equiv \bar{U}_{k}(\mathbf{r}, t)+\bar{U}_{\phi}(\mathbf{r}, t)
$$

and

$$
\bar{U}_{k}(\mathbf{r}, t)=\frac{m}{2 n} \int\left|\frac{\mathbf{p}}{m}-\mathbf{v}_{\mathbf{0}}\right|^{2} f_{1}(\mathbf{r}, \mathbf{p}, t) d \mathbf{p}
$$


is the average kinetic energy per molecule relative to $\mathbf{v}_{0}$, and

$$
\bar{U}_{\phi}(\mathbf{r}, t)=\frac{1}{2} \int \phi_{i n t}(\mathbf{R}) n_{2}(\mathbf{r}, \mathbf{R}, t) d \mathbf{R}
$$

is the intermolecular interaction potential energy average per molecule.

The energy flux vector relative to the mass average velocity, or differential heat, also has two contributions

$$
\mathbf{q}=\mathbf{q}_{k}+\mathbf{q}_{\phi}
$$

where

$$
\begin{gathered}
\mathbf{q}_{k} \equiv \frac{1}{2 m^{2}} \int f_{1}(\mathbf{r}, \mathbf{p}, t)\left|\mathbf{p}-m \mathbf{v}_{0}\right|^{2}\left(\mathbf{p}-m \mathbf{v}_{0}\right) d \mathbf{p} \\
\mathbf{q}_{\phi} \equiv \frac{1}{2 m} \int\left[\phi_{\text {int }}(R) \mathbf{I}-\frac{\mathbf{R} \mathbf{R}}{R} \frac{\partial \phi_{\text {int }}(R)}{\partial R}\right] \\
\cdot\left[\tilde{\mathbf{j}}_{1}^{(2)}(\mathbf{r}, \mathbf{R}, t)-m \mathbf{v}_{0} n^{2} \tilde{g}(\mathbf{r}, \mathbf{R}, t)\right] d \mathbf{R}
\end{gathered}
$$

and the two-particle function $\mathbf{j}_{1}^{(2)}\left(\mathbf{r}_{i}, \mathbf{r}_{j}, t\right)$ is defined as

$$
\mathbf{j}_{1}^{(2)}\left(\mathbf{r}_{i}, \mathbf{r}_{j}, t\right) \equiv \iint f_{2}\left(\mathbf{r}_{i}, \mathbf{r}_{j}, \mathbf{p}_{i}, \mathbf{p}_{j}\right) \mathbf{p}_{i} d \mathbf{p}_{i} d \mathbf{p}_{j}
$$

Finally, the continuity and momentum conservation equations can be eliminated from Equation (15) to equivalently express the general differential energy balance equation as

$$
n \frac{\partial}{\partial t} \bar{U}+n \mathbf{v}_{0} \cdot \frac{\partial}{\partial \mathbf{r}} \bar{U}=-\left(\frac{\partial}{\partial \mathbf{r}} \cdot \mathbf{q}\right)-\left(\mathbf{P}: \frac{\partial \mathbf{v}_{0}}{\partial \mathbf{r}}\right)
$$

where we have assumed that $\phi_{\text {ext }}$ is time independent for the sake of simplicity. Equation (23) is a celebrated form in energy transport phenomena, where the left-hand side relates time and space changes in the internal energy per molecule $\bar{U}$. On the righthand side of Equation (23), the first and second terms are the spatial gradients in the relative energy flux vector (differential heat) and part of differential work, respectively. Note that: in Equation (23) denotes the double dot tensor product operation. Looking at Equations (20) and (21), it is seen that differential heat q physically is the transfer of relative molecular kinetic energy and intermolecular interaction energies. It is unfortunate that the term "heat transfer" has persisted for so many years, since (differential) heat by definition is the transfer of energy across a (differential) boundary relative to the bulk flow; heat is never "transferred" - only energy is transferred.

\section{Results}

\subsection{Nonequilibrium Entropy Conservation}

Following Irving and Kirkwood's (IK) paradigm in development of the transport theory for mass, momentum, and energy, the nonequilibrium entropy conservation equation can be obtained by introducing a dynamic variable $\alpha$, which is defined here in terms of the Green z-functions, as [4]

$$
\alpha=-k_{B} \sum_{s}\left[\frac{N !}{s !(N-s) !}\right] \delta\left(\mathbf{r}_{j}-\mathbf{r}\right) z_{s}\left(\mathbf{r}^{s}, \mathbf{p}^{s}, t\right)
$$

where $k_{B}$ is Boltzmann's constant and the sum is over the set of molecules $s$ and $j$ is any molecule in the set $s$. Following Green [5], $z_{s}$ is the natural logarithm of an $s^{\text {th }}$ ordered, normalized density function that depends on the multi-particle expansion method [5,6]. Specifically,

$$
\begin{gathered}
z_{1}=\ln \left(\hbar^{3} f_{1}\right) \\
z_{2}=\ln \left[f_{2}\left(\mathbf{r}_{1}, \mathbf{r}_{2}, \mathbf{p}_{1}, \mathbf{p}_{2}, t\right) / f_{1}\left(\mathbf{r}_{1}, \mathbf{p}_{1}, t\right) f_{1}\left(\mathbf{r}_{2}, \mathbf{p}_{2}, t\right)\right]
\end{gathered}
$$


etc., for the so-called Kirkwood superposition approximation. Note that Equation (24) is a slightly simpler version of our previous definition [4] where it suffices for $\mathbf{r}_{j}$ in Equation (24) to be any molecule in the set $s$.

The phase-space average of $\alpha,\langle\alpha\rangle$, is computed by integrating $\alpha$ over all phasespace coordinates as

$$
<\alpha>=n \bar{S}_{B G}=-k_{B} \frac{1}{N !} \int \alpha f_{N} d \mathbf{r}^{N} d \mathbf{p}^{N}=-k_{B} \sum_{s} \frac{1}{s !} \iint \delta\left(\mathbf{r}_{j}-\mathbf{r}\right) f_{s} z_{s} d \mathbf{r}^{s} d \mathbf{p}^{s}
$$

where we have used the short-hand notation for differentials $\left(d \mathbf{r}^{N}=d \mathbf{r}_{1} d \mathbf{r}_{2} \cdot d \mathbf{r}_{N}\right.$, etc.) This average has been termed the "Boltzmann-Gibbs" entropy since it links Boltzmann's and Gibbs' definitions [3], where $\bar{S}_{B G}$ is the entropy per molecule and $n$ is the local molecular number density. For the lowest set $s=1$, we recover Boltzmann's definition [7]

$$
n \bar{S}_{1}(\mathbf{r}, t)=-k_{B} \int f_{1}\left(\mathbf{r}, \mathbf{p}^{\prime}, t\right) \ln \left(\hbar^{3} f_{1}\right) d \mathbf{p}^{\prime}
$$

where $\hbar$ is Planck's constant Extensions to other orders can also be shown. Specifically, for the set $\mathrm{s}=2$, we have

$$
n \bar{S}_{B G}=n \bar{S}_{1}+n \bar{S}_{2}
$$

where $n \bar{S}_{1}$ is given by Equation (28) and

$$
n \bar{S}_{2}(\mathbf{r}, t)=-\frac{k_{B}}{2 !} \iint f_{2}\left(\mathbf{r}, \mathbf{r}_{2}, \mathbf{p}_{1}, \mathbf{p}_{2}, t\right) z_{2}\left(\mathbf{r}, \mathbf{r}_{2}, \mathbf{p}_{1}, \mathbf{p}_{2}, t\right) d \mathbf{r}_{2} d \mathbf{p}_{1} d \mathbf{p}_{2}
$$

Note that various multiparticle expansions for the s-order density functions have been presented and reviewed by Singer [6]. By definition, entropy in these expressions represents the relative uncertainty in finding the group of $s$ molecules in the set of $N$ molecules. Typically, the lowest set $s=1$ corresponds to dilute gases, the set $s=2$ to dense gases, and so-forth for higher order sets.

Now, to obtain the entropy conservation equation following the IK approach given above for mass, momentum, and energy, we can multiply Equation (1) by

$$
-k_{B} \sum_{s} \frac{1}{(N-s) ! s !} z_{s} \delta\left(\mathbf{r}_{j}-\mathbf{r}\right),
$$

where $z_{1}, z_{2}, .$. are given by Equations (25) and (26), etc., and integrating over all $\left(\mathbf{r}^{N}, \mathbf{p}^{N}\right)$ space gives the following entropy conservation equation $[4,8]$

$$
n \frac{\partial \bar{S}_{B G}}{\partial t}+n \mathbf{v}_{0} \cdot \frac{\partial \bar{S}_{B G}}{\partial \mathbf{r}}=-\frac{\partial}{\partial \mathbf{r}} \cdot \mathbf{s}+n \bar{s}_{g}
$$

where the entropy flux vector $\mathbf{s}$ is defined according to

$$
\mathbf{s}(\mathbf{r}, t) \equiv-k_{B} \sum_{s} \frac{1}{s !} \iint \frac{\mathbf{p}_{1}^{\prime}}{m} f_{s}\left(\mathbf{r}, \mathbf{r}_{2}, . ., \mathbf{r}_{s}, \mathbf{p}_{1}, \mathbf{p}_{2}, . ., \mathbf{p}_{s}, t\right) z_{s}\left(\mathbf{r}, \mathbf{r}_{2}, . ., \mathbf{r}_{s}, \mathbf{p}_{1}, \mathbf{p}_{2}, . ., \mathbf{p}_{s^{\prime}}, t\right) d \mathbf{r}^{s-1} d \mathbf{p}^{s}
$$

which is the entropy flux relative to $\mathbf{v}_{0}$, where $\mathbf{p}_{1}^{\prime} / m=\mathbf{p}_{1} / m-\mathbf{v}_{0}$.

The last term on the right-hand side of Equation (31) is called the entropy generation term, specifically

$$
n \bar{s}_{g} \equiv-k_{B} \sum_{i=1}^{N} \iint\left[\sum_{k \neq i}^{N} \frac{\partial \phi\left(\mathbf{r}_{i, k}\right)}{\partial \mathbf{r}_{i}} \cdot \frac{\partial f_{N}}{\partial \mathbf{p}_{i}}\right]\left[\sum_{s} \frac{1}{(N-s) ! s !} z_{s} \delta\left(\mathbf{r}_{j}-\mathbf{r}\right)\right] d \mathbf{r}^{N} d \mathbf{p}^{N}
$$

Expanding the set $s$, the entropy generation term can thus be written generally as

$$
n \bar{s}_{g} \equiv n \bar{s}_{g_{1}}+n \bar{s}_{g_{2}}+n \bar{s}_{g_{3}}+\cdots
$$


where for the lowest set $s=1$, we obtain the well-known Boltzmann's entropy generation term $[7,9]$,

$$
n \bar{s}_{g 1}(\mathbf{r}, t)=k_{B} \iint \ln \left[\hbar^{3} f_{1}\left(\mathbf{r}, \mathbf{p}_{1}, t\right)\right]\left[\frac{\partial \Phi\left(\mathbf{r}_{2}, \mathbf{r}\right)}{\partial \mathbf{r}_{2}} \cdot \frac{\partial f_{2}\left(\mathbf{r}, \mathbf{r}_{2}, \mathbf{p}_{1}, \mathbf{p}_{2}, t\right)}{\partial \mathbf{p}_{1}}\right] d \mathbf{r}_{2} d \mathbf{p}_{1} \mathbf{p}_{2}
$$

and Newton's Third Law has been used in obtaining Equation (35). Higher orders can also be obtained straightforwardly.

In order to proceed further with elaboration of the above relations for entropy flux and generation, specific asymptotic expansions are needed $[4,8]$. For example, it can be formally shown that the entropy flux $\mathbf{s}=\mathbf{q} / T$, which confirms the well-known phenomenological result $[4,8]$. These expansions also verify that the entropy generation terms are always positive, thus providing a molecular basis for the Second Law [4,8], although the exact molecular theoretic origins of the Second Law outside of willful approximations [2,3] remains elusive.

The general forms of the balance equations given here are sufficient for us to examine simplified forms of the entropy conservation alongside their mass, momentum, and energy counterparts to help ensure consistency in their combined, practical use.

\subsection{Simplified Forms of the Mass, Momentum, Energy and Entropy Balance Equations Local Equilibrium Flows}

The first common, simplified form of the transport equations are for so-called local equilibrium flows. For local equilibrium flows, the density function $f_{N}$ in the Liouville equation can be shown to follow a particular, locally canonical equilibrium form [10]

$$
f_{N}^{l o c e q}=\frac{n_{N}\left(\mathbf{r}^{N}, t\right)}{[2 \pi m k T(\mathbf{r}, t)]^{\frac{3 N}{2}}} \exp \left\{-\sum_{j=1}^{3 N} \frac{\left[\mathbf{p}_{j}-\mathbf{v}_{0}(\mathbf{r}, t)\right]^{2}}{2 m k T(\mathbf{r}, t)}\right\}
$$

leading to [10]

$$
\left.\begin{array}{c}
\mathbf{P}=p \mathbf{I} \\
\mathbf{q}=0 \\
\mathbf{s}=0 \\
\bar{s}_{g}=0
\end{array}\right\} \text { local equilibrium approximation }
$$

where, in Equation (36), $n_{N}\left(\mathbf{r}^{N}, t\right)$ is the $N$-particle equilibrium configurational density function, which is now possibly dependent on space and time through local values of number density $n(\mathbf{r}, t)$, velocity $\mathbf{v}_{0}(\mathbf{r}, t)$, and /or temperature $T(\mathbf{r}, t)$. Note that temperature here is defined by the classical definition as a quantity proportional to the average molecular velocity relative to the bulk flow, i.e.,

$$
\frac{\frac{1}{2 m} \int p_{k}^{\prime 2} f_{N} d \mathbf{p}^{N}}{\int f_{N} d \mathbf{p}^{N}}=\frac{3}{2} k T
$$

where $p_{k}^{\prime}$ is the momentum of any kth molecule relative to the local bulk flow. Using the relationships of Equation (37), the balance equations then simplify to

$$
\begin{aligned}
& \text { mass : } \frac{\partial \rho}{\partial t}+\frac{\partial}{\partial \mathbf{r}} \cdot\left(\rho \mathbf{v}_{0}\right)=0 \\
& \text { momentum }: \frac{\partial \mathbf{v}_{0}}{\partial t}+\left(\mathbf{v}_{0} \cdot \frac{\partial}{\partial \mathbf{r}}\right) \mathbf{v}_{0}=-\frac{1}{\rho} \frac{\partial p}{\partial \mathbf{r}}+\frac{\mathbf{F}_{e x t}}{m} \\
& \text { energy }: n \frac{\partial \bar{U}}{\partial t}+n \mathbf{v}_{0} \cdot \frac{\partial \bar{U}}{\partial \mathbf{r}}=-p\left(\frac{\partial}{\partial \mathbf{r}} \cdot \mathbf{v}_{0}\right) \\
& \text { entropy : } n \frac{\partial \bar{S}_{B G}}{\partial t}+n \mathbf{v}_{0} \cdot \frac{\partial \bar{S}_{B G}}{\partial \mathbf{r}}=0
\end{aligned}
$$


and $p$ follows from a local thermodynamic equation of state (e.g., a van der Waals equation of state), $p=p(n, T)$, and $\mathrm{n}$ and $\mathrm{T}$ are local values of the number density and temperature, respectively. Thus, from Equation (42), we see that local equilibrium flows are locally isoentropic flows where entropy stays constant along fluid streamlines.

\subsection{Reversible Flows}

Reversible flows are defined as flows that are inviscid and do not generate entropy. However, these flows do include heat.

$$
\left.\begin{array}{c}
\mathbf{P}=p \mathbf{I} \\
\mathbf{s}=\mathbf{q} / T \\
\bar{s}_{g}=0
\end{array}\right\} \text { local reversible }
$$

Thus, for locally reversible flows, we have the set of transport equations:

$$
\begin{gathered}
\text { mass : } \frac{\partial \rho}{\partial t}+\frac{\partial}{\partial \mathbf{r}} \cdot\left(\rho \mathbf{v}_{0}\right)=0 \\
\text { momentum : } \frac{\partial \mathbf{v}_{0}}{\partial t}+\left(\mathbf{v}_{0} \cdot \frac{\partial}{\partial \mathbf{r}}\right) \mathbf{v}_{0}=-\frac{1}{\rho} \frac{\partial p}{\partial \mathbf{r}}+\frac{\mathbf{F}_{e x t}}{m} \\
\text { energy }: n \frac{\partial \bar{U}}{\partial t}+n \mathbf{v}_{0} \cdot \frac{\partial \bar{U}}{\partial \mathbf{r}}=-p\left(\frac{\partial}{\partial \mathbf{r}} \cdot \mathbf{v}_{0}\right)-\frac{\partial}{\partial \mathbf{r}} \cdot \mathbf{q} \\
\text { entropy : } n \frac{\partial \bar{S}_{B G}}{\partial t}+n \mathbf{v}_{0} \cdot \frac{\partial \bar{S}_{B G}}{\partial \mathbf{r}}=-\frac{\partial}{\partial \mathbf{r}} \cdot \frac{\mathbf{q}}{T}
\end{gathered}
$$

Any other simplified forms of the transport equations, such as isothermal and incompressible, will follow by the same holistic analysis of the complete set of transport equations.

Another important aspect of the complete and consistent set of transport equations is that we can combine the basis thermodynamic functions $p(\bar{V}, T), \bar{U}(\bar{V}, T)$, and $S(\bar{V}, T)$, as done in global equilibrium systems, to obtain derived transport equations that may simplify applications. For example, we can write a completely general nonequilibrium Gibbs' transport equation beginning with $\bar{G}=\bar{U}-T \bar{S}+p \bar{V}$, using the total derivative $D / D t=\partial / \partial t+\mathbf{v} \cdot \partial / \partial \mathbf{r}$, and Equations (8), (9), (23), and (31), to obtain

$$
\frac{D \bar{G}}{D t}=\bar{S} \frac{D T}{D t}+\bar{V} \frac{D p}{D t}
$$

where $\bar{V}=1 / n$. This derived, Gibbs' transport equation may be of used for a nonequilibrium phase change which takes place locally at constant $\mathrm{T}$ and $\mathrm{p}$. In general, these type of derived equations are, of course, not independent of the basis transport equations, but may simplify analyses and applications.

\section{Discussion and Conclusions}

Entropy conservation or the Second Law plays a critical role is setting limits on heat and work that are possible in any given system. It is imperative then that entropy conservation equations be developed consistently across the transport equations since these involve a variety of approximations necessary in practice. Irving and Kirkwood's molecular theoretical derivations of the conservations equations of mass, momentum, and energy represent the most rigorous classical theory of transport phenomena and serves as a basis for more complex systems. We have developed an entropy conservation equation or the Second Law following the IK method. We formally derive simplified forms of the complete set of transport equations that will be useful in applications, including local equilibrium flows and reversible flows. We show that local equilibrium flows are also locally isoentropic flows. In addition, the complete set of transport equations allows us to develop derived transport equations, such as a nonequilibrium Gibbs' transport equation derived here, which may be useful, for example, in flows with phase change. 
Funding: There was no external funding associated with this research.

Institutional Review Board Statement: Not Applicable.

Informed Consent Statement: Not Applicable.

Data Availability Statement: The data presented in this study are available on request from the corresponding author.

Conflicts of Interest: There are no conflicts of interest to declare.

\section{References}

1. Irving, J.H.; Kirkwood, J.G. The Statistical Mechanical Theory of Transport Processes. IV. The Equations of Hydrodynamics. J. Chem. Phys. 1950, 18, 817-829. [CrossRef]

2. Jaynes, E.T. Information Theory and Statistical Mechanics. Phys. Rev. 1957, 106, 620-630. [CrossRef]

3. Jaynes, E.T. Information Theory and Statistical Mechanics. II. Phys. Rev. 1957, 108, 171-190. [CrossRef]

4. Peters, M. Generalized Entropy Generation Expressions in Gases. Entropy 2019, 21, 330. [CrossRef] [PubMed]

5. Green, H.S. The Molecular Theory of Fluids; OCLC: 877638975; Dover Publications Constable: New York, NY, USA; London, UK, 1969.

6. Singer, A. Maximum entropy formulation of the Kirkwood superposition approximation. J. Chem. Phys. 2004, 121, 3657-3666. [CrossRef] [PubMed]

7. Hirschfelder, J.O.; Curtiss, C.F.; Bird, R.B. Molecular Theory of Gases and Liquids; Structure of Matter Series, OCLC: 70387122; Wiley: New York, NY, USA, 1964.

8. Peters, M.H. Completing Irving and Kirkwood's Molecular Theory of Transport Processes: Nonequilibrium Entropy Conservation. Ind. Eng. Chem. Res. 2009, 48, 166-171. [CrossRef]

9. Chapman, S.; Cowling, T.G. The Mathematical Theory of Non-Uniform Gases: An Account of the Kinetic Theory of Viscosity, Thermal Conduction, and Diffusion in Gases, 3rd ed.; Cambridge Mathematical Library, Cambridge University Press: Cambridge, UK, 1990.

10. Peters, M.H. Molecular Thermodynamics and Transport Phenomena: Complexities of Scales in Time and Space; McGraw-Hill: New York, NY, USA, 2005. 